SLAC-PUB-10504

SU-ITP-04/25

\title{
A Note on Mirror Symmetry for Manifolds with $\operatorname{Spin}(7)$ Holonomy
}

\author{
Wu-yen Chuang \\ SLAC, and Department of Physics \\ Stanford University, Stanford, CA 94305 \\ wychuang@itp.stanford.edu
}

\begin{abstract}
Starting from the superconformal algebras associated with $G_{2}$ manifolds, I extend the algebra to the manifolds with spin(7) holonomy. I show how the mirror symmetry in manifolds with spin(7) holonomy arises as the automorphism in the extended sperconformal algebra. The automorphism is realized as 14 kinds of T-dualities on the supersymmetric $T^{4}$ toroidal fibrations. One class of Joyce's orbifolds are pairwise identified under the symmetry.
\end{abstract}

Work supported in part by the Department of Energy Contract DE-AC03-76SF00515

Stanford Linear Accelerator Center, Stanford University, Stanford, CA 94309 


\section{Introduction}

Mirror symmetry is a beautiful subject both in physics and mathematics. It was first conjectured in [1] that there exists a symmetry which exchanges the complex moduli on one manifold with the Kahler moduli on the dual manifold when we consider the string worldsheet propagation on Calabi-Yau target spaces. The symmetry arises in the sense that the resulting physical spectra of the mirror pair are isomorphic. This requires the

Betti numbers of the CY mirror pair satisfy the condition $b_{p, q}(M)=b_{d-p, q}(\tilde{M})$. It was also shown that mirror symmetry could determine non-perturbative effects of worldsheet instantons by counting the number of holomorphic curves in Calabi-Yau spaces [2]. Those who are interested in various aspects of mirror symmetry are referred to [3].

In [4], Strominger, Yau and Zaslow (SYZ) argued that the mirror transformation is equivalent to T-duality on the supersymmetric $T^{3}$ fibration in the Calabi-Yau manifolds, by considering the mirror BPS soliton spectra in two theories (IIA/IIB). Some concrete mirror pairs of certain toroidal orbifolds with discrete torsion can be found in [5], where the mirror symmetry is indeed realized as T-duality on toroidal $T^{3}$ fibration in the orbifolds. And these examples involving the changes of discrete torsion are related to the main goal of this paper.

In [6][7], Acharya discussed the existence of the mirror symmetry in IIA/IIB string theory compactified on manifolds with exceptional holonomy and argued how the discrete torsion trasforms under the $T^{4}$ T-duality. In [8], the authors gave some concrete mirror pairs among Joyce's orbifolds with $G_{2}$ holonomy, which are built from resolving or deforming $T^{7} / Z_{2}^{3}$ orbifolds. They also identified the mirror symmetry as an automorphism in the extended superconformal algebra on manifolds with $G_{2}$ holonomy.

Motivated by these known results, I generalize the chiral superconformal algebra to the manifolds with spin(7) holonomy, and identify the corresponding automorphism in the algebra as a combination of a T-duality in 8-direction and a generalized $G_{2}$ mirror transformation or a combination of two distinct $G_{2}$ mirror transformations. The automorphism could also be understood as T-duality on the supersymmetric $T^{4}$ fibrations. In order to make the automorphism clearer, I give an example of one class of Joyce's spin(7) manifolds with discrete torsion. The 14 kinds of $T^{4} \mathrm{~T}$-dualities are classified into two categories, one of which does flip the discrete torsion and hence lead to a topologically different Joyce's orbifold and the other does not.

The paper is presented as follows. In section 2 I review the mirror symmetry of Calabi-Yau and $G_{2}$ manifolds both from the viewpoint of the conformal field theory and 
the T-duality. In section 3 I will give the construction of $\operatorname{spin}(7)$ extended superconformal algebra and identify the automorphism in it as 14 kinds of T-dualities and classify them into two kinds as mentioned above. Section 4 is conclusion and some suggestion for future study.

\section{Mirror symmetry for $\mathrm{CY}$ and $G_{2}$ manifolds}

In this section I will give a short review of mirror symmetry on Calabi-Yau orbifolds $\left(T^{6} / Z_{2}^{2}\right)$ and Joyce's $G_{2}$ manifolds [8][9][10].

\subsection{Mirror symmetry of Calabi-Yau threefolds}

The generators of the $N=2$ superconformal algebra for string propagation on CalabiYau target-space are the stress energy tensor $T_{C Y}$, two supercurrent $G_{C Y}, G_{C Y}^{\prime}$ and the $\mathrm{U}(1)$ current $J_{C Y}$, along with a complex current $\Omega_{C Y}$ of conformal weight $3 / 2$ constructed from the worldsheet fermions and its superpartner $\Psi_{C Y}$.

In $T^{6} / Z_{2}^{2}$ orbifolds, they can be expressed as, [8][11][12]

$$
\begin{aligned}
T_{C Y} & =\frac{1}{2} \sum_{j=1}^{6}: \partial x^{j} \partial x^{j}:-\frac{1}{2} \sum_{j=1}^{6}: \psi^{j} \partial \psi^{j}:, \\
G_{C Y} & =\sum_{j=1}^{6}: \psi^{j} \partial x^{j}:, G_{C Y}^{\prime}=\sum_{j=1}^{3}\left(\psi^{2 j-1} \partial x^{2 j}-\psi^{2 j} \partial x^{2 j-1}\right), J_{C Y}=\sum_{j=1}^{3} \psi^{2 j-1} \psi^{2 j}, \\
\Omega_{C Y} & =\psi^{1} \psi^{3} \psi^{5}-\psi^{1} \psi^{4} \psi^{6}-\psi^{2} \psi^{3} \psi^{6}-\psi^{2} \psi^{4} \psi^{5}+i\left(\psi^{1} \psi^{3} \psi^{6}+\psi^{1} \psi^{4} \psi^{5}+\psi^{2} \psi^{3} \psi^{5}-\psi^{2} \psi^{4} \psi^{6}\right), \\
\Psi_{C Y} & :=\left\{G_{C Y}, \Omega_{C Y}\right\} .
\end{aligned}
$$

There exists an automorphism in the superconformal algebra or OPE, which leave invariant the $N=1$ superconformal subalgebra generated by $T_{C Y}$ and $G_{C Y}$.

$$
G_{C Y}^{\prime} \rightarrow-G_{C Y}^{\prime}, \quad J \rightarrow-J, \quad \Omega \rightarrow \Omega^{*}, \quad \Psi \rightarrow \Psi^{*}
$$

Calabi-Yau mirror symmetry is to apply the above automorphism to one of the chiralities of the algebra, for instance, $\tilde{G}_{C Y}^{\prime}, \tilde{J}_{C Y}, \tilde{\Omega}_{C Y}$, and $\tilde{\Psi}_{C Y}$. Recall that the T-duality in $i$ th direction will leave $\partial x_{i}$ and $\psi_{i}$ invariant but reverse $\bar{\partial} x_{i}$ and $\tilde{\psi}_{i}$. Therefore, we can easily see that the T-duality on $T^{3}$ fibrations in the following directions ( which appear in the indices of $\Omega_{C Y}$ ) also generates the mirror symmetry. 


$$
\{(1,3,5),(1,4,6),(2,3,6),(2,4,5),(1,3,6),(1,4,5),(2,3,5),(2,4,6)\}
$$

Some concrete examples of these T-dualities acting on the $T^{3}$ fibration and changing the discrete torsion can be found in [5][8].

\subsection{Compact orbifolds with $G_{2}$ holonomy}

In this and the following sections, I will first give an example of Joyce's orbifolds which were constructed by disingularising $T^{7} / Z_{2}^{3}$ and how the choices in resolving (deforming) the singularities can result in topologically different spaces. After that, I will write down the $G_{2}$ extended chiral superconformal algebra and look for the automorphism in it. We will see that applying the automorphism transformation to one of two chiralities is equivalent to applying a T-duality on certain $T^{3}$ toroidal fibration.

Consider the orbifolds of $T^{7} / \Gamma$, where $x_{i}=x_{i}+1$ and $\Gamma$ is generated by three $Z_{2},[9]$

$$
\begin{aligned}
& \alpha=\left(-x_{1},-x_{2},-x_{3},-x_{4}, x_{5}, x_{6}, x_{7}\right), \\
& \beta=\left(-x_{1}, 1 / 2-x_{2}, x_{3}, x_{4},-x_{5},-x_{6},-x_{7}\right), \\
& \gamma=\left(-x_{1}, x_{2},-x_{3}, x_{4},-x_{5}, x_{6},-x_{7}\right) .
\end{aligned}
$$

In order to desingularize the orbifolds, one has to know, for instance, how the $16 \alpha$ fixed $T^{3} s$ get identified under the group generated by $\beta$ and $\gamma$. What we found in this example is the $16 T^{3} s$ fixed by $\alpha$ or $\beta$ are reduced to 4 orbits of order 4 by the free-acting of the $\langle\beta, \gamma\rangle$ or $\langle\gamma, \alpha\rangle$. In the $\gamma$-fixed $T^{3}$ sector, the group $\langle\alpha, \beta\rangle$ only reduce 16 $T^{3}$ to 8 orbits of order 2 since $\alpha \beta$ acts trivially on them.

The choices of blowing-up or deforming also come from this $\gamma$-fixed sector. From a discrete torsion analysis based on the requirement of modular invariance [8], we know that blowing-up (deforming) corresponds to discrete torsion in the $\gamma$-fixed sector $\epsilon_{\gamma ; \tilde{f}}=1(-1)$ and the even (odd) $\alpha \beta$ parity. By virtue of the correspondence between the RR ground states and the cohomology, we can write down the RR ground states in $\gamma$-fixed sector.

For $\alpha \beta$ parity even case, we have,

$$
\begin{aligned}
& \epsilon_{\gamma ; \tilde{f}}=1, \\
& |0,0 ; \tilde{f}\rangle_{\gamma}, \psi^{2+}|0,0 ; \tilde{f}\rangle_{\gamma}, \psi^{4+} \psi^{6+}|0,0 ; \tilde{f}\rangle_{\gamma}, \psi^{2+} \psi^{4+} \psi^{6+}|0,0 ; \tilde{f}\rangle_{\gamma},
\end{aligned}
$$

where $\tilde{f}=1, . .8$ labelling the $\gamma$-fixed points after $\alpha$ or $\beta$ identification. 
For $\alpha \beta$ parity odd case, the $\mathrm{RR}$ ground states are,

$$
\begin{aligned}
& \epsilon_{\gamma ; \tilde{f}}=-1, \\
& \psi^{4+}|0,0 ; \tilde{f}\rangle_{\gamma}, \psi^{6+}|0,0 ; \tilde{f}\rangle_{\gamma}, \psi^{2+} \psi^{4+}|0,0 ; \tilde{f}\rangle_{\gamma}, \psi^{2+} \psi^{6+}|0,0 ; \tilde{f}\rangle_{\gamma}
\end{aligned}
$$

One should regard $|0,0 ; \tilde{f}\rangle_{\gamma}$ as the harmonic two form associated with the exceptional divisors of the blowing-up (deformation). Therefore, blowing-up contributes 1 to $b_{2}$ and 1 to $b_{3}$ while the deformation increases $b_{3}$ by 2 .

For the RR ground states in $\gamma$-fixed sector, the operation $\alpha \beta$ reverses the 4 th and 6th directions. Therefore, we can express it as,

$$
\alpha \beta=\frac{1}{4} \psi_{0}^{4} \psi_{0}^{6} \tilde{\psi}_{0}^{4} \tilde{\psi}_{0}^{6} \epsilon_{\gamma ; \tilde{f}} .
$$

We denote $X_{l}$ Joyce's manifold with $l$ blow-ups and $8-l$ deformations. After summing up all Betti numbers from various sectors, we have,

$$
\left(b_{0}, \ldots, b_{7}\right)=(1,0,8+l, 47-l, 47-l, 8+l, 0,1) .
$$

\section{3. $G_{2}$ extended superconformal algebra}

The algebra on manifolds with $G_{2}$ holonomy is generated by appending a spin $3 / 2$ operator $\Phi_{G_{2}}$ and its superpartner $X_{G 2}$ to the $N=1$ superconformal subalgebra spanned by $T_{G_{2}}$ and $G_{G_{2}}$ [11][13]. In our basis of coordinates, they are,

$$
\begin{aligned}
T_{G_{2}} & =\frac{1}{2} \sum_{j=1}^{7}: \partial x^{j} \partial x^{j}:-\frac{1}{2} \sum_{j=1}^{7}: \psi^{j} \partial \psi^{j}:, \quad G_{G_{2}}=\sum_{j=1}^{6}: \psi^{j} \partial x^{j}: \\
\Phi_{G_{2}} & =\psi^{1} \psi^{3} \psi^{6}+\psi^{1} \psi^{4} \psi^{5}+\psi^{2} \psi^{3} \psi^{5}-\psi^{2} \psi^{4} \psi^{6}+\psi^{1} \psi^{2} \psi^{7}+\psi^{3} \psi^{4} \psi^{7}+\psi^{5} \psi^{6} \psi^{7} \\
X_{G 2} & =-\psi^{2} \psi^{4} \psi^{5} \psi^{7}-\psi^{2} \psi^{3} \psi^{6} \psi^{7}-\psi^{1} \psi^{4} \psi^{6} \psi^{7}+\psi^{1} \psi^{3} \psi^{5} \psi^{7}-\psi^{3} \psi^{4} \psi^{5} \psi^{6} \\
& -\psi^{1} \psi^{2} \psi^{5} \psi^{6}-\psi^{1} \psi^{2} \psi^{3} \psi^{4}-\frac{1}{2} \sum_{j=1}^{7}: \psi^{j} \partial \psi^{j}:
\end{aligned}
$$

The extended superconformal algebra has one obvious automorphism [11][13].

$$
\Phi_{G 2} \rightarrow-\Phi_{G_{2}} ; \quad K_{G 2} \rightarrow-K_{G 2} ; T_{G 2}, G_{G 2}, X_{G 2}, M_{G 2} \text { unchanged }
$$


If the $G_{2}$ manifolds are of the form $\left(C Y_{3} \times S^{1}\right) / Z^{2}$ as the Joyce $G_{2}$ manifolds, we can also reformulate the superconformal generators in terms of the Calabi-Yau ones.

$$
\begin{aligned}
T_{G_{2}} & =T_{C Y}+\frac{1}{2}: \partial x^{7} \partial x^{7}:-\frac{1}{2}: \psi^{7} \partial \psi^{7}:, \quad G_{G_{2}}=G_{C Y}+: \psi^{7} \partial x^{7}: \\
\Phi_{G_{2}} & =\operatorname{Im}\left(\Omega_{C Y}\right)+: J_{C Y} \psi^{7}: \\
X_{G_{2}} & =: \operatorname{Re}\left(\Omega_{C Y}\right) \psi^{7}:+\frac{1}{2}: J_{C Y} J_{C Y}:-\frac{1}{2}: \psi^{7} \partial \psi^{7}: \\
K_{G 2} & =\operatorname{Im}\left(\Psi_{C Y}\right)+: J_{C Y} \partial x^{7}:+: G_{C Y}^{\prime} \psi^{7}:, \\
M_{G 2} & =: \operatorname{Re}\left(\Psi_{C Y}\right) \psi^{7}:-: \operatorname{Re}\left(\Omega_{C Y}\right) \partial x^{7}:+: \partial x^{7} \partial \psi^{7}:+: J_{C Y} G_{C Y}^{\prime}:-\frac{1}{2} \partial G_{C Y} .
\end{aligned}
$$

Similarly, the generalized mirror symmetry for manifolds with $G_{2}$ holonomy is to apply the above automorphism to one of the two chiralities. On the other hand, the T-duality in the following $\left(i_{1}, i_{2}, i_{3}\right)$ directions can obviously realize the automorphism.

$$
\begin{aligned}
& \left(i_{1}, i_{2}, i_{3}\right) \in I_{3}^{+} \cup I_{3}^{-} \\
& I_{3}^{+}=\{(2,4,6),(2,3,5),(1,2,7)\} \\
& I_{3}^{-}=\{(1,3,6),(1,4,5),(3,4,7),(5,6,7)\}
\end{aligned}
$$

If we combine any two different T-dualities listed above, we obtain another set of T-dualities acting on toroidal $T^{4}$, which also leave the extended chiral algebra invariant. Hence, they are mirror symmetry which take IIA (IIB) to IIA (IIB).

$$
\begin{aligned}
& \left(i_{1}, i_{2}, i_{3}, i_{4}\right) \in I_{4}^{+} \cup I_{4}^{-}, \\
& I_{4}^{+}=\{(1,3,5,7),(1,4,6,7),(3,4,5,6)\}, \\
& I_{4}^{-}=\{(2,4,5,7),(2,3,6,7),(1,2,5,6),(1,2,3,4)\} .
\end{aligned}
$$

Recall that T-duality in $i$ th direction will give $\tilde{\psi}_{0}^{i}$ a minus sign. It's not hard to see that $I_{3}^{+}\left(I_{4}^{+}\right)$does not change the discrete torsion while $I_{3}^{-}\left(I_{4}^{-}\right)$does. We can summarize the action of the T-dualities as follows.

$$
\begin{aligned}
& I I A(I I B) / X_{l} \longleftrightarrow I I B(I I A) / X_{8-l}, \quad \text { under } I_{3}^{-} \\
& I I A(I I B) / X_{l} \longleftrightarrow I I A(I I B) / X_{l}, \quad \text { under } I_{3}^{+} .
\end{aligned}
$$




\section{Mirror symmetry for $\operatorname{spin}(7)$ manifolds}

\subsection{Joyce's construction of spin(7) manifolds}

There are many known examples of Joyce spin(7) orbifolds [14]. For simplicity, I will take one orbifold for example in which we have choices in desingularizing $T^{8} / Z_{2}^{4}$ as before. The generators are,

$$
\begin{aligned}
& \alpha=\left(-x_{1},-x_{2},-x_{3},-x_{4}, x_{5}, x_{6}, x_{7}, x_{8}\right), \\
& \beta=\left(x_{1}, x_{2}, x_{3}, x_{4},-x_{5},-x_{6},-x_{7},-x_{8}\right), \\
& \gamma=\left(1 / 2-x_{1},-x_{2}, x_{3}, x_{4}, 1 / 2-x_{5},-x_{6}, x_{7}, x_{8}\right), \\
& \delta=\left(-x_{1}, x_{2}, 1 / 2-x_{3}, x_{4}, 1 / 2-x_{5}, x_{6}, 1 / 2-x_{7}, x_{8}\right) .
\end{aligned}
$$

Again, the periodicity of $x_{i}$ is unity. In general, the singularities arises in five different types and the corresponding desingularization is following.

Type(1): increase $b_{2}$ by $1, b_{3}$ by $4, b_{4+}$ by 3 , and $b_{4-}$ by 3 . The singularity type is $T^{4} \times\left(B_{\epsilon}^{4} /\{ \pm 1\}\right)$, where $B_{\epsilon}^{4}$ is defined as an open ball of radius $\epsilon$ about 0 in $R^{4}$.

Type(2): increase $b_{2}$ by $1, b_{4+}$ by 3 , and $b_{4-}$ by 3 . The singularity if of the form $\left(T^{4} /\{ \pm 1\} \times\left(B_{\epsilon}^{4} /\{ \pm 1\}\right)\right.$.

Type(3): increase $b_{4+}$ by 1 . The singularity is $\left(B_{\epsilon}^{4} /\{ \pm 1\} \times\left(B_{\epsilon}^{4} /\{ \pm 1\}\right)\right.$.

Type $(4 \mathrm{~A})$ increase $b_{2}$ by $1, b_{3}$ by $2, b_{4+}$ by 1 , and $b_{4-}$ by 1 .

Type(4B) increase $b_{3}$ by $2, b_{4+}$ by 2 , and $b_{4-}$ by 2 .

The singularity of type(4) is an isometric involution $\sigma$ of $T^{4} \times\left(B_{\epsilon}^{4} /\{ \pm 1\}\right)$, where $\sigma=\left(1 / 2+x_{1}, x_{2},-x_{3},-x_{4}, y_{1}, y_{2},-y_{3},-y_{4}\right)$. Namely, the singular set is isomorphic to $\left(T^{4} \times\left(B_{\epsilon}^{4} /\{ \pm 1\}\right)\right) /<\sigma>$.

Type(5A) increase $b_{2}$ by $1, b_{4+}$ by 1 , and $b_{4-}$ by 1 .

Type(5B) increase $b_{4+}$ by 2 , and $b_{4-}$ by 2 .

The singularity of type(5) is isomorphic to $\left(T^{4} /\{ \pm 1\} \times B_{\epsilon}^{4} /\{ \pm 1\}\right) /\langle\sigma\rangle$.

As a result, one found the singular set of this orbifold contains 2 type(1), 8 type(2), 64 type(3) and 4 type(4). If we choose to have $j$ type(4A) and $4-j$ type(4B) and add up all the Betti numbers in the twisted sectors as well as the untwisted sector, we have the Joyce's manifolds $Y_{j}$ with

$$
\begin{gathered}
b_{2}=10+j, b_{3}=16, b_{4+}=109-j, b_{4-}=45-j, \quad j=0, \ldots, 4 \\
\hat{A}=\frac{1}{24}\left(-1+b_{1}-b_{2}+b_{3}+b_{4+}-2 b_{4-}\right)=1 .
\end{gathered}
$$


In fact, the 4 type(4) singularities come from $16 \gamma$-fixed $T^{4} \mathrm{~s}$. Notice that $\alpha \delta$ acts trivially on these $T^{4} \mathrm{~s}$ and the group elements $\alpha, \beta, \alpha \beta$, and $\beta \delta$ act freely on them and reduce the number of $T^{4} \mathrm{~s}$ to be 4 . Therefore, we have $\mathrm{RR}$ ground states $|0,0 ; \tilde{f}=1,2,3,4\rangle_{\gamma}$ corresponding to the harmonic two forms of the exceptional divisors. Similarly, the $\alpha \delta$ parity of $|0,0 ; \tilde{f}\rangle_{\gamma}$ is also given by the discrete torsion $\epsilon_{\gamma, \tilde{f}}$. Since the action of $\alpha \delta$ inverses direction 4 and 7 , we can construct RR ground states accordingly as follows.

For $\alpha \delta$ parity even case, we have,

$$
\begin{aligned}
& \epsilon_{\gamma ; \tilde{f}}=1, \\
& |0,0 ; \tilde{f}\rangle_{\gamma}, \psi^{3+}|0,0 ; \tilde{f}\rangle_{\gamma}, \psi^{8+}|0,0 ; \tilde{f}\rangle_{\gamma}, \psi^{3+} \psi^{8+}|0,0 ; \tilde{f}\rangle_{\gamma}, \psi^{4+} \psi^{7+}|0,0 ; \tilde{f}\rangle_{\gamma}, \\
& \psi^{3+} \psi^{4+} \psi^{7+}|0,0 ; \tilde{f}\rangle_{\gamma}, \psi^{4+} \psi^{7+} \psi^{8+}|0,0 ; \tilde{f}\rangle_{\gamma}, \psi^{3+} \psi^{4+} \psi^{7+} \psi^{8+}|0,0 ; \tilde{f}\rangle_{\gamma} .
\end{aligned}
$$

For $\alpha \delta$ parity odd case, the RR ground states are,

$$
\begin{aligned}
& \epsilon_{\gamma ; \tilde{f}}=-1, \\
& \psi^{4+}|0,0 ; \tilde{f}\rangle_{\gamma}, \psi^{7+}|0,0 ; \tilde{f}\rangle_{\gamma}, \\
& \psi^{3+} \psi^{4+}|0,0 ; \tilde{f}\rangle_{\gamma}, \psi^{3+} \psi^{7+}|0,0 ; \tilde{f}\rangle_{\gamma}, \psi^{4+} \psi^{8+}|0,0 ; \tilde{f}\rangle_{\gamma}, \psi^{7+} \psi^{8+}|0,0 ; \tilde{f}\rangle_{\gamma}, \\
& \psi^{3+} \psi^{4+} \psi^{8+}|0,0 ; \tilde{f}\rangle_{\gamma}, \psi^{3+} \psi^{7+} \psi^{8+}|0,0 ; \tilde{f}\rangle_{\gamma} .
\end{aligned}
$$

Obviously, we obtain $\Delta b_{2}=1, \Delta b_{3}=2, \Delta b_{4}=2$ in parity even case, and $\Delta b_{3}=$ $2, \Delta b_{4}=4$ in parity odd case, which agrees with the mathematical analysis in [14].

\section{2. $\operatorname{spin}(7)$ extended superconformal algebra}

Consider a direct product space $M \times S^{1}$, where $M$ is a manifold with $G_{2}$ holonomy. It is always possible to define a $\operatorname{spin}(7)$ structure. And the Cayley 4 -form $\phi_{4}$ in this manifold with $\operatorname{spin}(7)$ structure can be written as,

$$
\phi_{4}=* \phi_{3}+\phi_{3} \wedge d x^{8}
$$

where $\phi_{3}$ is the calibrated three form in the $G_{2}$ manifold.

It is true that in the example in the previous section $T^{7} /\langle\alpha, \gamma, \delta\rangle$ gives rise to a Joyce's 7-manifold of $G_{2}$ holonomy, if we forget about 8-direction. In fact, we can have a more generic statement which is $T^{7} /\langle\alpha, \gamma, \delta\rangle$ is always a manifold with $G_{2}$ holonomy for any choices of the constants $c_{i}$ and $d_{i}[15]$, where 


$$
\begin{aligned}
\alpha & =\left(-x_{1},-x_{2},-x_{3},-x_{4}, x_{5}, x_{6}, x_{7}\right), \\
\gamma & =\left(c_{1}-x_{1}, c_{2}-x_{2}, x_{3}, x_{4}, c_{5}-x_{5}, c_{6}-x_{6}, x_{7}\right), \\
\delta & =\left(c_{1}-x_{1}, x_{2}, c_{3}-x_{3}, x_{4}, c_{5}-x_{5}, x_{6}, c_{7}-x_{7}\right) .
\end{aligned}
$$

If we reformulate the action of $\beta$ in the previous section, we will find that $\beta$ acts as,

$$
\beta: x^{8} \rightarrow-x^{8}, \beta^{*}\left(\phi_{3}\right)=-\phi_{3}, \beta^{*}\left(* \phi_{3}\right)=* \phi_{3} .
$$

In this example, $\beta$ indeed turns the $\operatorname{spin}(7)$ structure into the $\operatorname{spin}(7)$ holonomy. However, it is not clear that we can always form manifolds with spin(7) holonomy by modding out this kind of $Z_{2}$ involution on $G_{2} \times S^{1}$.

Therefore, at least in Joyce's orbifolds, the relation (3.6) enables us to write down the expression of the stress energy tensor $T_{\operatorname{spin}(7)}$ and the supercurrent $G_{\operatorname{spin}(7)}$ in terms of the corresponding quantities in $G_{2}$ manifolds [13].

$$
\begin{aligned}
T_{\operatorname{spin}(7)} & =T_{G_{2}}+\frac{1}{2}: \partial x^{8} \partial x^{8}:-\frac{1}{2}: \psi^{8} \partial \psi^{8}: \\
G_{\operatorname{spin}(7)} & =G_{G_{2}}+: \psi^{8} \partial x^{8}: \\
X_{\operatorname{spin}(7)} & =X_{G_{2}}+\Phi_{G_{2}} \psi^{8}+\frac{1}{2} \psi^{8} \partial \psi^{8} \\
M_{\operatorname{spin}(7)} & =\left[G_{\operatorname{spin}(7)}, X_{\operatorname{spin}(7)}\right] \\
& =\partial x^{8} \Phi_{G_{2}}-K_{G 2} \psi^{8}-M_{G_{2}}+\frac{1}{2} \partial^{2} x^{8} \psi^{8}-\frac{1}{2} \partial x^{8} \partial \psi^{8} .
\end{aligned}
$$

From these generators for the extended supercomformal algebra, it is not difficult to see that the combination of the $G_{2}$ automorphism (2.10) and the T-duality in 8-direction is an automorphism in the algebra. In addition, the T-duality in (2.13) is also an automorphism in the algebra. Therefore, we have a list of $14 \mathrm{~T}$-dualities on $T^{4}$ toroidal fibrations which generate the mirror symmetry,

$$
\begin{aligned}
& \{(2,4,6,8),(2,3,5,8),(1,2,7,8),(1,3,6,8),(1,4,5,8),(3,4,7,8),(5,6,7,8), \\
& (1,2,5,7),(1,4,6,7),(3,4,5,6),(2,4,5,7),(2,3,6,7),(1,2,5,6),(1,2,3,4)\} .
\end{aligned}
$$

The first line consists of T-dualities in directions in (2.12) and 8-direction. The second line is the same as the directions listed in (2.13). In this spin(7) case, we don't have the similar relation like (2.10). Therefore, in order to visualize the automorphism in the algebra, we have to express the $\operatorname{spin}(7)$ generators and the algebra in terms of $G_{2}$ generators and 
construct our desirable mirror transformation from $G_{2}$ automorphism $(2.10)(2.12)(2.13)$. Finally, the expression of $\alpha \delta$ in $\gamma$-fixed sector is,

$$
\alpha \delta=\frac{1}{4} \psi_{0}^{4} \psi_{0}^{7} \tilde{\psi}_{0}^{4} \tilde{\psi}_{0}^{7} \epsilon_{\gamma ; \tilde{f}}
$$

By the same reasoning, the 14 T-dualities are divided into two sets $J_{4}^{ \pm}$. Their action is also summarized as follows.

$$
\begin{aligned}
&\left(i_{1}, i_{2}, i_{3}, i_{4}\right) \in J_{4}^{+} \cup J_{4}^{-} \\
& J_{4}^{+}=\{(2,3,5,8),(1,3,6,8),(3,4,7,8),(1,4,6,7),(2,4,5,7),(1,2,5,6)\} \\
& J_{4}^{-}=\{(2,4,6,8),(1,2,7,8),(1,4,5,8),(5,6,7,8),(1,2,5,7),(3,4,5,6), \\
& \\
&\quad(2,3,6,7),(1,2,3,4)\} .
\end{aligned}
$$

$$
\begin{aligned}
& I I A(I I B) / Y_{j} \longleftrightarrow I I A(I I B) / Y_{4-j}, \quad \text { under } J_{4}^{-} \\
& I I A(I I B) / Y_{j} \longleftrightarrow I I A(I I B) / Y_{j}, \quad \text { under } J_{4}^{+} .
\end{aligned}
$$

\section{Conclusion}

In this paper I have generalized the construction of [8] to the Joyce's manifolds with $\operatorname{spin}(7)$ holonomy and shown how the mirror symmetry is realized in the superconformal algebra as a combination of a T-duality in 8-direction and a $G_{2}$ mirror symmetry transformation, or a combination of 2 distinct $G_{2}$ mirror transformations. The spin(7) mirror transformation contains 14 different kinds of T-dualities on the $T^{4}$ fibrations. By an analysis on the change of discrete torsion, one can classify these 14 T-dualities into 2 kinds, one of which changes the discrete torsion and the other does not.

In [16], the authors completed a cycle of the dualities by explicitly performing the T-duality on $T^{3}$ fibration and a $G_{2}$ flop in M-theory. It would be interesting to generalize the computation to a duality cycle involving $\operatorname{spin}(7)$ and $G_{2}$ manifolds and understand how the generalized mirror symmetry lies in this picture [17].

In order to understand the $G_{2} / \operatorname{spin}(7)$ mirror symmetry better, one may try to Tdualize the known various non-compact metric solutions with $G_{2} / \operatorname{spin}(7)$ holonomy [18] and see how they are connected through mirror symmetry. In the Calabi-Yau case, NS-NS fluxes can turn the CY target space into half-flat [19]. The generalized mirror symmetry for $G_{2}$ and $\operatorname{spin}(7)$ in the presence of the background fluxes also begs some further study. 
Finally, it would also be interesting to see how we can fit the $G_{2}$ or spin(7) mirror symmetry into the correspondence of heterotic $\left(G_{2}\right) / \operatorname{IIA}\left(G_{2}\right.$ orientifold $) / \mathrm{M}$-theory $(\operatorname{spin}(7))$ [20].

\section{Acknowledgements}

I would like to thank Peter Kaste for his useful comment on the draft. The research of WYC is supported by supported by the U.S. Department of Energy under contract number DE-AC03-76SF00515. 


\section{References}

[1] W. Lerche, C. Vafa and N. P. Warner, "Chiral Rings In N=2 Superconformal Theories," Nucl. Phys. B 324, 427 (1989).

[2] P. Candelas, X. C. De La Ossa, P. S. Green and L. Parkes, "A Pair Of Calabi-Yau Manifolds As An Exactly Soluble Superconformal Theory," Nucl. Phys. B 359, 21 (1991).

[3] D. A. Cox and S. Katz, "Mirror Symmetry And Algebraic Geometry," American Mathematical Society, (September 1, 1999)

[4] A. Strominger, S. T. Yau and E. Zaslow, "Mirror symmetry is T-duality," Nucl. Phys. B 479, 243 (1996) [arXiv:hep-th/9606040].

[5] C. Vafa and E. Witten, "On orbifolds with discrete torsion," J. Geom. Phys. 15, 189 (1995) [arXiv:hep-th/9409188].

[6] B. S. Acharya, "On mirror symmetry for manifolds of exceptional holonomy," Nucl. Phys. B 524, 269 (1998) [arXiv:hep-th/9707186].

[7] B. S. Acharya, "Exceptional mirror symmetry" Winter School on Mirror Symmetry, Vector Bundles and Lagrangian Submanifolds (Cambridge, MA, 1999), P.1-P.14, AMS/IP Stud. Adv. Math., 23.

[8] M. R. Gaberdiel and P. Kaste, "Generalised discrete torsion and mirror symmetry for G(2) manifolds," arXiv:hep-th/0401125.

[9] D. D. Joyce, "Compact Riemannian 7-manifolds with holonomy $G_{2}$. I," J. Diff. Geom. 43 (1996) 291

[10] D. D. Joyce, "Compact Riemannian 7-manifolds with holonomy $G_{2}$. II," J. Diff. Geom. 43 (1996) 329.

[11] J. M. Figueroa-O'Farrill, "A note on the extended superconformal algebras associated with manifolds of exceptional holonomy," Phys. Lett. B 392, 77 (1997) [arXiv:hepth/9609113].

[12] S. Odake, "Extension Of $N=2$ Superconformal Algebra And Calabi-Yau Compactification," Mod. Phys. Lett. A 4, 557 (1989).

[13] S. L. Shatashvili and C. Vafa, "Superstrings And Manifold Of Exceptional Holonomy," arXiv:hep-th/9407025.

[14] D. D. Joyce, "Compact 8-manifolds with holonomy Spin(7)," Inv. Math. 123 (1996) 507.

[15] B. S. Acharya, "N=1 M-theory-Heterotic Duality in Three Dimensions and Joyce Manifolds," arXiv:hep-th/9604133.

[16] M. Becker, K. Dasgupta, A. Knauf and R. Tatar, "Geometric transitions, flops and non-Kaehler manifolds. I," arXiv:hep-th/0403288.

[17] S. Gukov, J. Sparks and D. Tong, "Conifold transitions and five-brane condensation 
in M-theory on Spin(7) manifolds," Class. Quant. Grav. 20, 665 (2003) [arXiv:hepth/0207244].

[18] M. Cvetic, G. W. Gibbons, H. Lu and C. N. Pope, "Cohomogeneity one manifolds of Spin(7) and G(2) holonomy," Phys. Rev. D 65, 106004 (2002) [arXiv:hep-th/0108245].

[19] S. Gurrieri, J. Louis, A. Micu and D. Waldram, "Mirror symmetry in generalized Calabi-Yau compactifications," Nucl. Phys. B 654, 61 (2003) [arXiv:hep-th/0211102].

[20] J. Majumder, "Type IIA orientifold limit of M-theory on compact Joyce 8-manifold of Spin(7)-holonomy," JHEP 0201, 048 (2002) [arXiv:hep-th/0109076]. 